\title{
What If We Administered the "Wrong" Inventory? The Prediction of Scores on Personality Research Form Scales from Those on the California Psychological Inventory, and Vice Versa
}

\author{
Lewis R. Goldberg \\ University of Oregon
}

Equations are presented for estimating the scores on each of 20 PRF scales and each of 19 CPI scales from the other inventory. Estimates of the cross-validity of these inter-inventory prediction equations ranged from about .40 to approximately .80 . Scores on the typical CPI scale are predicted reasonably well by one or two PRF content scales, plus the Desirability scale. While scores on most PRF scales can be equally well estimated by a few CPI scales, one PRF scale--Sentience-appears to be unpredictable from the CPI.

One of the major stumbling blocks in the development of a cumulative science of individual differences has been the recent proliferation of personality scales and inventories (Goldberg, 1971) and the tendency of investigators to include in their own research only their favorite measure. As a consequence, it has been difficult to align the findings from different investigations along any consistent set of dimensions. Moreover, many classic assessment projects, including some costly longitudinal studies as well as some important assessments of highly gifted and creative individuals, were forced to include measures which are already becoming

APPLIED PSYCHOLOGICAL MEASUREMENT

Vol. 1, No. 3 Summer 1977 pp. 339-354

(C) Copuright 1977 West Publishing Co. obsolete, with the result that their rich findings may have no direct concordance with those from later studies using more contemporary instruments. To help solve this problem, we need a set of cross-inventory prediction equations that permit the estimation of scale scores on any one inventory from those on a number of others. This report is the first in a series from a project aimed at the development of precisely such a translation base.

While it is not uncommon to find reports of the correlations between the scales from two inventories or even of factor analyses based on such inter-inventory correlations, investigators have only recently begun to explore the problem of inter-inventory scale predictability via multiple regression procedures. Inter-inventory regression equations have now been reported between the 16PF and the MMPI (Cattell \& Bolton, 1969; Cattell, Eber, \& Tatsuoka, 1970), between the 16PF and the CPI (Campbell \& Chun, 1977), and between the CPI and the SVIB (Johnson, Flammer, \& Nelson, 1975). In addition, investigators have recently used such methodology for predicting Rotter's Locus of Control scale from the CPI (Gough, 1974), as well as from the MMPI and the CPI combined (Scott \& Severance, 1975). For a re- 
view of the major studies of inter-inventory correspondence, see Campbell \& Chun (1977).

In the present study, the scales from one of the most popular inventories developed in the past, Gough's California Psychological Inventory (CPI), constructed during the early 1950 s, were used to predict those in Jackson's Personality Research Form (PRF), constructed a decade later, and vice versa. These two inventories, each of which was intended to provide a reasonably comprehensive mapping of personality structure, were devised by radically different strategies of personality scale construction (see Goldberg, 1972a). Eleven of the 18 original CPI scales were constructed by the external ("empirical") strategy of scale construction. Later, a series of 11 -scale "inventories" was developed from the CPI item pool, each "inventory" constructed by a different strategy (Hase \& Goldberg, 1967). For a recent critique of the CPI, see Goldberg (1972b); for a detailed review of research on the CPI scales, see Megargee (1972).

In contrast to the CPI, whose construction was stimulated by the success of the external strategy in the development of the MMPI, the construction of Jackson's PRF was stimulated by the wave of research and theorizing about the importance of response sets and styles in previous inventories (see Edwards, 1957; Jackson, 1960; Jackson \& Messick, 1958). As a consequence, the PRF was specifically developed by a variety of procedures aimed at the attenuation or control of stylistic response variance. For a discussion of these procedures, see Jackson (1970); for an evaluation of their success, see Anastasi (1972), Kelly (1972), Wiggins (1972), and Stricker (1974).

The original CPI scales were designed to measure "folk concepts" (e.g., dominance, sociability, responsibility, tolerance, flexibility). Later CPI scales (Hase \& Goldberg, 1967) included some designed to measure eleven of the 27 "manifest needs" posited by the personality theorist, Henry Murray (1938). Of the 22 PRF scales, 16 were designed to meas- ure such "manifest needs," while three more were constructed as measures of "general traits" in Murray's theoretical system.' Nine constructs are measured by scales in both inventories.

\section{Method}

\section{Subjects and Procedures}

The 315 students who enrolled in an introductory-level course, "Personality and Individual Differences," at the University of Oregon were given a wide variety of options for accruing course credits. One such option, completing one or more from a set of 12 personality inventories, was described in the following manner in the course syllabus:

While no one is obligated to complete any of the inventories, thinking about one's own personality in terms of these sorts of questions can be a very en riching experience. Moreover, if you are interested in learning about psychologists' attempts to measure personality traits, one important means to assess today's "state of the art" is by completing some currently popular inventories. All inventories may be completed either in class or at home (in some quiet and undisturbed hiding place).

Scores on the inventory scales will probably not be available at any time during the Quarter, due to the difficulties in scoring all of the test protocols; students interested in receiving their inventory scores may have to wait a year or two until the inventories are scored and nontechnical interpretations of the various personality scales are prepared. Therefore, you should elect to take the inventories solely for the experience itself, not

'For a complete list of Murray's constructs, and the scales from five recent inventories that have been targeted for each of them, see Goldberg (1971). 
necessarily for any score feedback you may ultimately receive.

In spite of the disclaimer about score feedback, the option of taking personality inventories turned out to be one of the most popular in the course, and many of the inventories were completed by well over 200 students.

For each inventory in turn, all response protocols were examined for evidence of possible invalidity by checking for $(a)$ response omission to any sizeable number of items, $(b)$ gross response imbalance (selection of primarily one response category), $(c)$ response inconsistency to repeated items (where available), and $(d)$ response deviance (selection of many highly implausible and statistically infrequent response options). After such potentially invalid protocols were eliminated from the sample, there typically remained about 150 subjects who had completed any particular pair of inventories.

In the case of the CPI and PRF, 154 students ( 85 women and 69 men) remained for analysis in the present study, after potentially invalid response protocols were removed from the sample, including those from subjects scoring higher than 2 on the "Infrequency" (In ) scale of the PRF or scoring lower than 21 on the "Commonality" $(\mathrm{Cm})$ scale of the CPI. Each student had completed the standard 480-item booklet for the CPI, plus the 440 items in either Form AA or BB of the PRF. Roughly half of the group responded to each of the two PRF forms. Since the means, standard deviations, and scale intercorrelations were virtually identical in the subsamples from each of these two parallel PRF forms, they were pooled for all analyses presented in this report.

\section{Analyses}

The standard 22 PRF scales and a set of 49 CPI scales ${ }^{2}$ were scored; these 71 scales are

${ }^{2}$ For the items, and keying direction, for each of these 49 CPI scales, see Appendix 1 in Megargee (1972). listed in Table 1. The scale abbreviations presented in Table 1 will be used throughout the remainder of this report.

Means, standard deviations, and intercorrelations among these 71 scales were separately computed in the male and female subsamples, and in the total sample. Subsequently, the total sample was randomly divided into two equal subsamples, each of size 77 , for a series of stepwise regression analyses, using a double crossvalidation design. Additional stepwise multiple regression analyses were carried out on the total sample, which also was used for the calculation of canonical correlations.

\section{Results and Discussion}

The means, standard deviations, and intercorrelations among the 22 PRF scales, separately computed in the female and male subsamples as well as in the total sample, are presented in Goldberg (1976). All mean scores in both subsamples fell within one standard deviation of those from the two normative samples used by Jackson to develop the PRF profile sheets, and most of the mean scores were precisely the same as those found in his normative samples. The primary difference between the present group and the normative ones was on the Autonomy scale, where the present group had somewhat higher mean scores. Scale intercorrelations were generally low and usually quite similar in magnitude in the two subsamples. While only $13 \%$ of the correlations in the female group and $9 \%$ in the male group were as high as .40 , a few were quite substantial [e.g., Achievement vs. Endurance (.74); Dominance vs. Exhibition (.64); Affiliation vs. Nurturance (60); Autonomy vs. Succorance (-.58); and Order vs. Cognitive Structure (.56)] .

The means, standard deviations, and intercorrelations among the 49 CPI scales in both subsamples and in the total group are presented in Goldberg (1976). Again, the mean scores for the 18 original scales in both subsamples fell within a standard deviation of those 


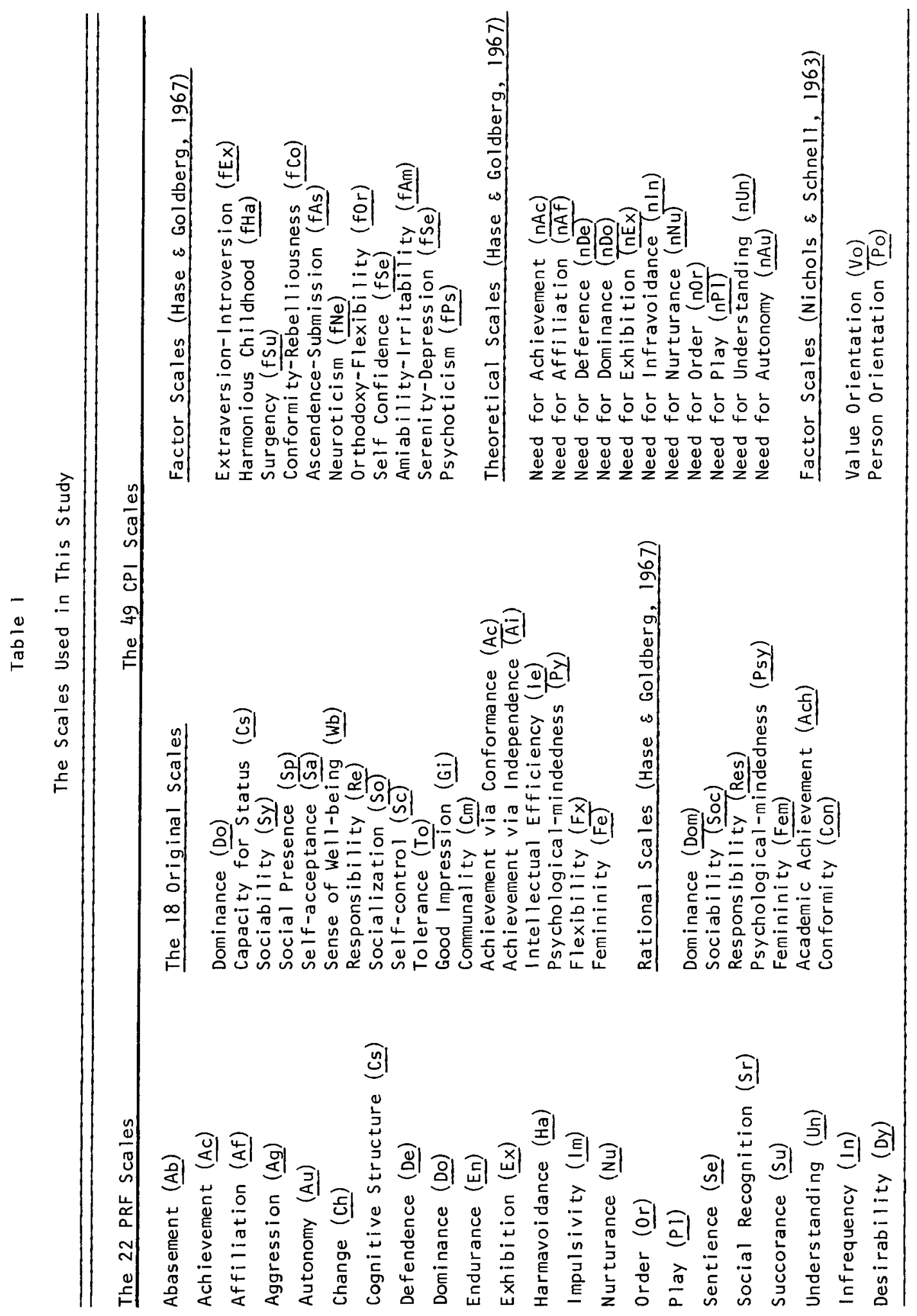


from the two normative samples used by Gough to develop the CPI profile sheets. In comparison to the normative samples, the present subsamples scored a bit higher on the Social Presence, Self-acceptance, Achievement via Independence, and Flexibility scales, and a trifle lower on most of the Class II scales (e.g., Responsibility, Self-control, and Good-impression).

As expected, CPI scale intercorrelations are often quite substantial, since no attempt has been made to keep them independent, and many pairs of scales include a large proportion of common items. For the 18 original scales, $36 \%$ of the correlations in the female group and $33 \%$ in the male group were at least .40 , and within the entire set of 49 scales there were numerous pairs with intercorrelations well above .70. As a consequence, it is important to realize that this set of $49 \mathrm{CPI}$ scales is quite redundant and that the number of relatively independent scales in the CPI set is certainly no higher than the 22 provided by the PRF.

The cross-inventory correlations for the male and female subsamples are presented in Goldberg (1976), while those for the total group are listed in Table 2 . Over $60 \%$ of the 49 CPI scales were correlated at least .40 with the PRF Desirability $(D y)$ scale; indeed, the scale developed to mark the first CPI common factor (Vo) correlated .69 with $D y$. The highest CPI correlate of $D y$, nonetheless, was the validity scale $W b(r=.72)$, which was constructed empirically to discriminate persons feigning neurosis from actual neurotic patients.

The degree of convergent and discriminant validity for the nine identically labeled scales in the two inventories was generally quite substantial. The convergent validity coefficients are listed here in descending order: Dominance (.80), Order (.74), Affiliation (.63), Understanding (.59), Achievement (.57), Exhibition (.56), Autonomy (.47), Play (.47), and Nurturance $(.28)$. Except for Nurturance, where the PRF measure was more highly related to the need for Affiliation ( $n A f$ ) scale on the CPI than to its namesake, corresponding scales were generally more highly correlated with each other than with any other scales. However, because this CPI scale set is so redundant, there were usually a half dozen or so CPI scales that correlated substantially with any single PRF scale; for example, high CPI correlates of $D o$ in the PRF include-in addition to $n D_{o}(.80)-D o m(.79)$, Do (.74), fAs (.80), and Po (.75).

\section{Canonical Correlations}

The nature of this redundancy may be clarified by reference to Table 3, which presents the canonical correlations between the two scale sets, and lists the scales that had substantial correlations with the canonical composites. Only the first seven of the 22 canonical correlations were significant by Bartlett's Test; the eighth coefficient had a probability value of .11 , the ninth of .47 , and the tenth of .79 . And, with only one exception (the PRF Sentience scale on the 14th canonical), none of the scales from either inventory correlated as highly as .40 with any beyond the first six composites.

The pattern of scale loadings on the canonical composites does not perfectly mirror the structure typically found when the scales in each inventory are factored alone (e.g., Megargee, 1972; Nesselroade \& Baltes, 1975; Stricker, 1974). The first canonical composite pulled together scales to form a broad Conscientiousness factor, loaded most highly by the Need for Order scales on the PRF $(r=.77)$ and the CPI $(r=.72)$. The second composite, which pulled together a set of Dominance and Assertiveness scales to form a broad Surgency factor, was similar to the second factor found when the CPI scales were factored alone; the marker for this CPI factor $(\mathrm{Po})$ correlated .60 with the composite. The third composite, which pulled together diverse measures of Sociability, was correlated most highly with the Need for Affiliation scales on the PRF $(r=.74)$ 


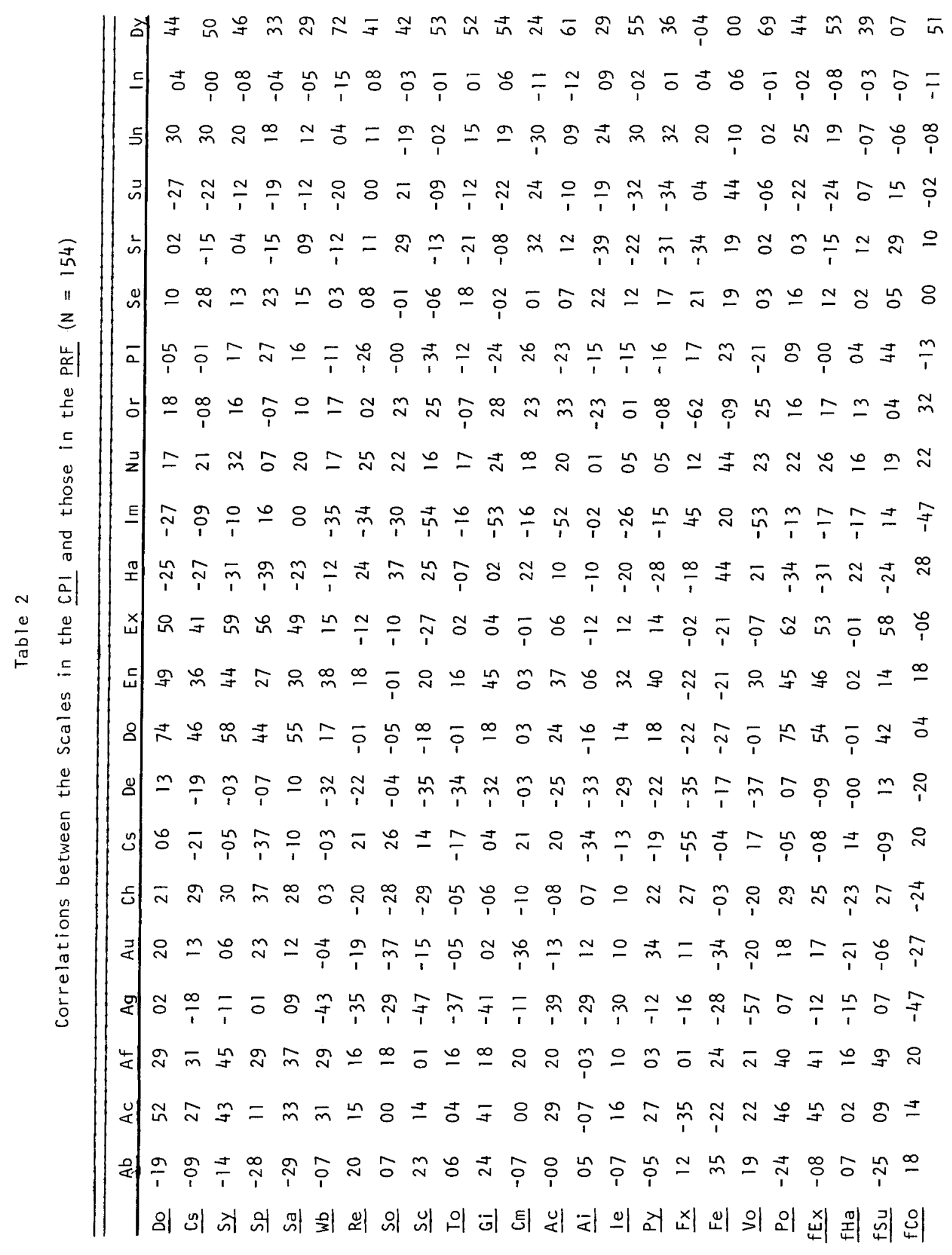




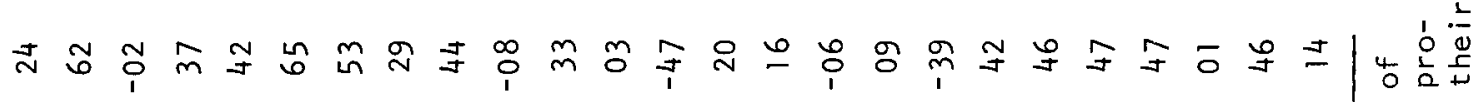
으응ำ

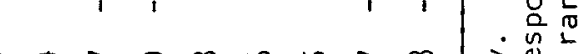
$N \cong \underset{1}{N} \vec{m}$ 음

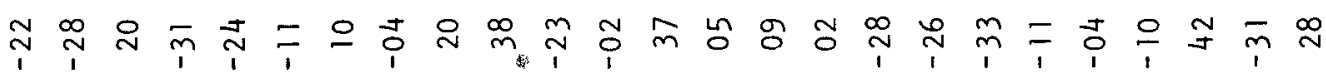

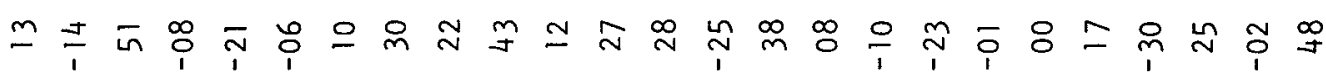

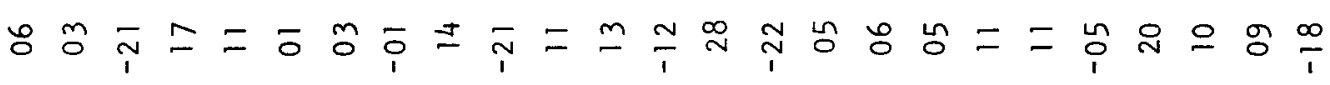
무윙 少

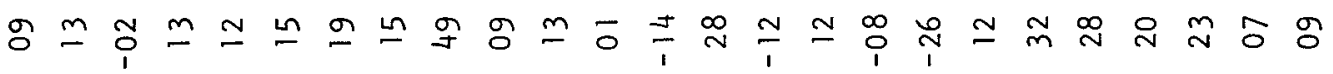

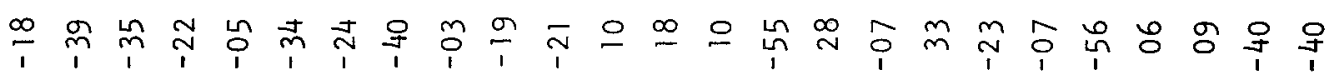

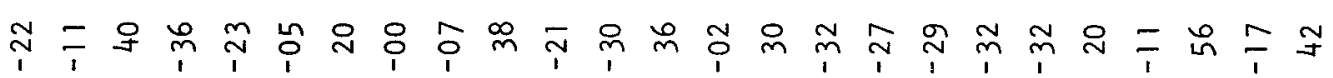

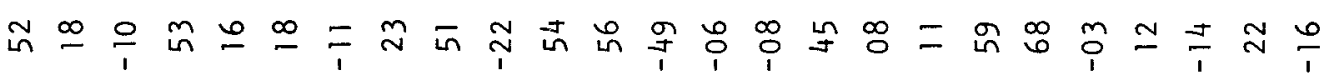

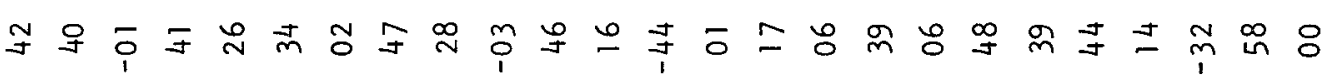

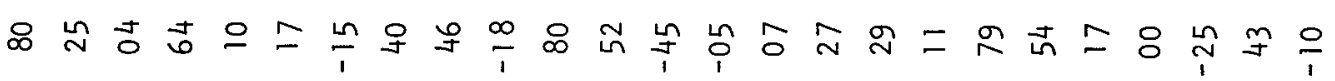

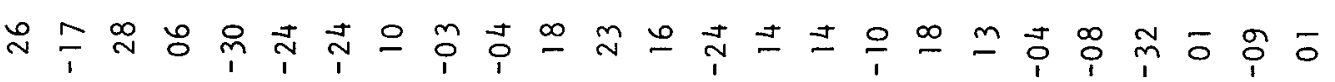
ํำ

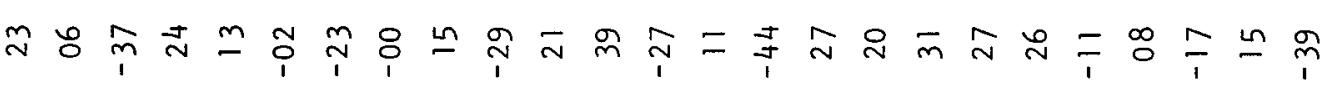

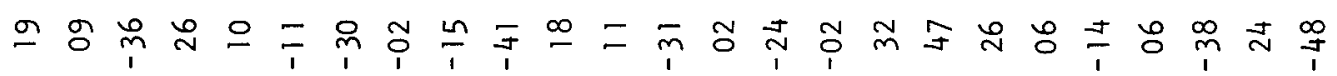

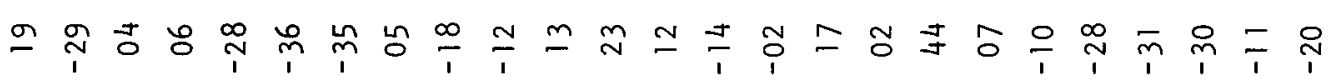

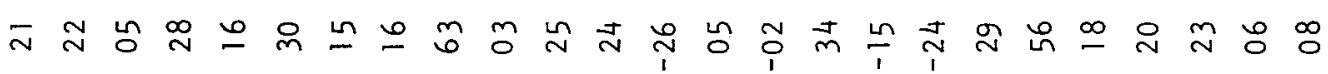

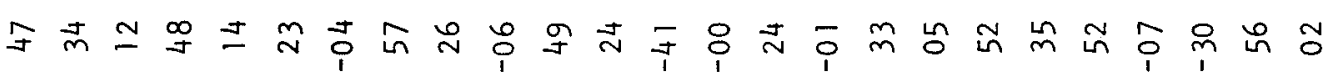

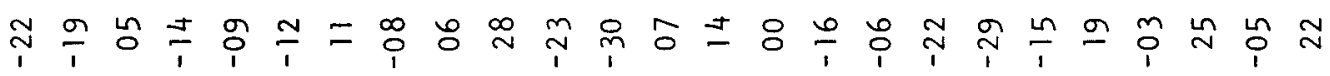

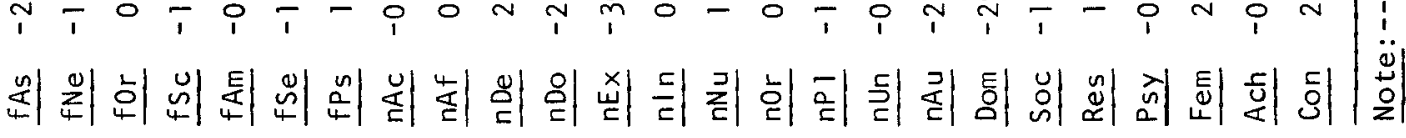
$\stackrel{0}{\geq} \div$ 讨茫 으는 in. 는 $1 \geq \stackrel{\text { in }}{1}$ $-\pi$ $\stackrel{0}{>} \div$

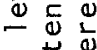
음 잉 문 它交市 ᄂก - U⿺辶万人 里员识 + in เ $0 \stackrel{0}{0}$ 오는든 立运 $=\frac{1}{0}$ 등 ᄂᄂ $>\mathbb{U}$ Un 牙\& ᄃ。 . $\frac{1}{0}$ in $\div \frac{1}{3} \ldots \pi$ 它至 in $\bar{n}$ 就 ᄂ $\frac{2}{0} \frac{1}{0}$

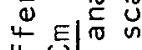
: ত - 尼 $\frac{0}{\circ}=$ 近员 $\frac{0}{\mathrm{E}} \bar{\sigma}$ $\left.\because \frac{1}{1}\right|_{\frac{1}{5}} ^{\text {in }} \frac{5}{3}$ ᄉ in 는 $\frac{\omega}{0}$ 든 今

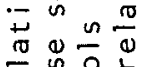

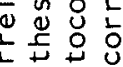

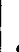




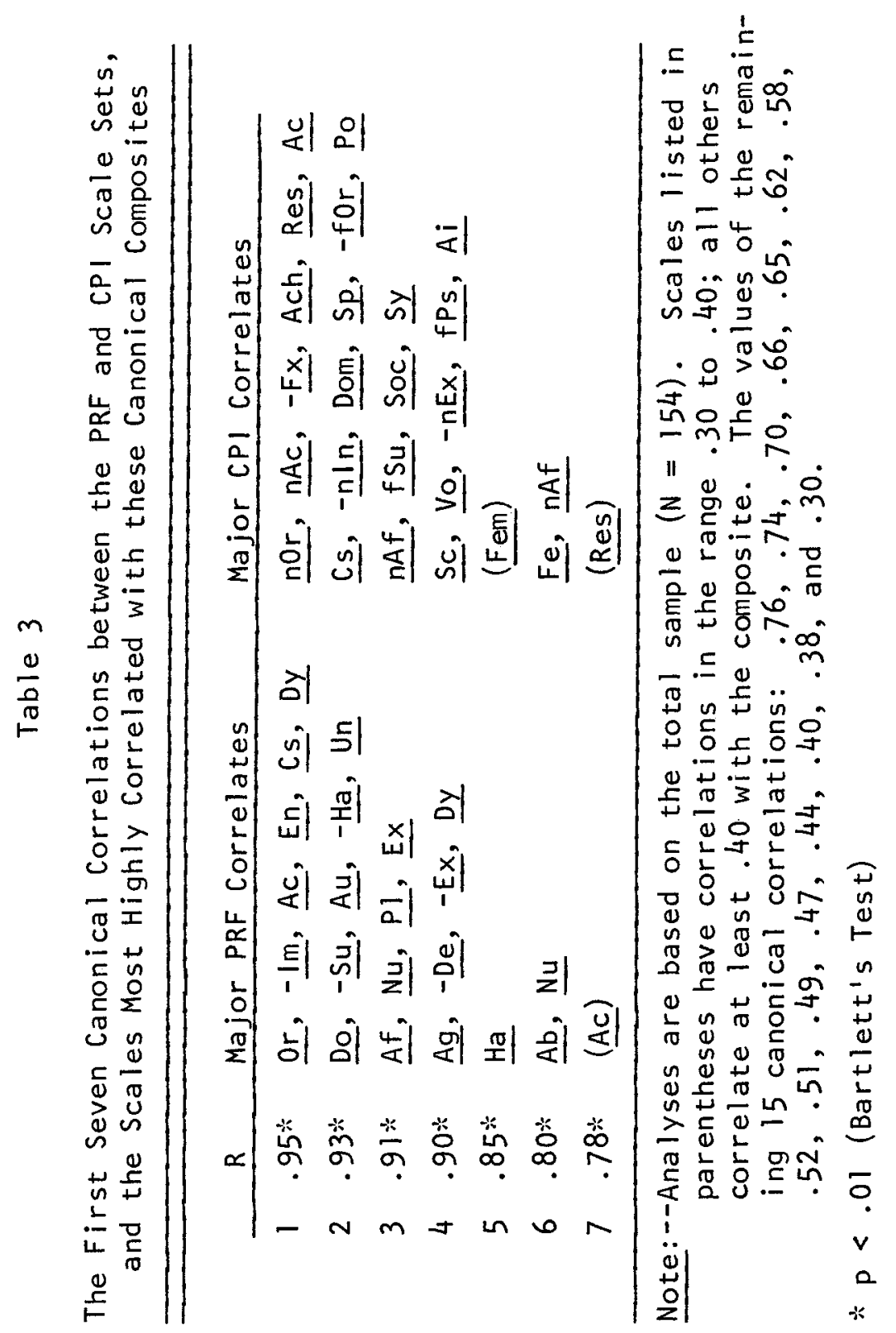


and the CPI ( $r=.69$ ). The fourth composite pitted those scales typically forming the first CPI factor ( Vo: $r=.70$ ) against the Need for Aggression ( $r=-.68$ ) scale on the PRF. The fifth composite linked the PRF Harmavoidance scale $(r=.44)$ and the rationally constructed CPI Femininity (Fem) scale $(r=.39)$. The sixth composite paired the PRF Abasement $(r=.54)$ and Nurturance $(r=.53)$ scales with the empirically constructed CPI Femininity $(F e)$ scale $(r=.53)$.

\section{Stepwise Regression Analyses: Cross-Validity Coefficients}

While there is already some evidence concerning the optimal number of predictors to include in regression equations for samples of roughly this size (Goldberg, 1972a), it should be instructive to replicate previous analyses using the present data set. Although additional predictors can never decrease the multiple correlation in the sample used to construct the regression equations, they can certainly serve to attenuate cross-validity in another sample. Table 4 presents the average cross-validity coefficients as a function of the number of scales included in the regression equation, when the set of 49 CPI scales was used as an initial pool to predict each of the PRF scales. These analyses are based on a double cross-validation design, the total sample having been randomly split into two subsamples, each of 77 students. The information presented in Table 4 will be used for two purposes: (a) to obtain a lower bound estimate of the correlation we may expect in new samples for each of the total-sample regression equations, and (b) to help determine the number of steps to be included in each of those total-sample analyses.

First, let us consider how accurately the regression equations are likely to generalize beyond the present sample. For two of the PRF scales (Do and $O r$ ), the CPI regression equations should predict their values about as well as their reliabilities permit, with estimated correlations of approximately .80 . At the other extreme, one PRF scale ( $S e$ ) seems to be completely unpredictable from the CPI. While only modest predictive accuracy may be anticipated for six PRF scales $(A b, A g, C h, D e, P l$, and $\mathrm{Su}$ )-their average correlations estimated around .45 -the remaining half of the cross-inventory equations should produce correlations of about .65 with the scales they are constructed to predict.

Because many of the CPI scales employed in this project are not routinely scored in the research of other investigators, various subsets of scales were used in analogous analyses so that the results could be compared with those based upon the total set. Employed in these comparisons, the results of which are presented in Goldberg (1976), were the following subsets of CPI scales: (a) the 18 original scales, (b) the 11 Theoretical scales, (c) the 11 Factor scales, (d) the 11 Rational scales, and (e) those five Rational scales identified in Goldberg (1972a) as being of particular predictive potency. A summary of all of these cross-validity analyses, presented in Table 5, shows that the average PRF scale was best predicted by a four or five scale composite selected from the entire 49 scale set. If only the 11 Rational scales (or, alternatively, only the 11 Theoretical scales) are scored, cross-validity is typically reduced only slightly. On the other hand, use of the 18 original scales or of the 11 Factor scales resulted in a somewhat more substantial loss in cross-validity.

The analogous cross-validity coefficients are presented in Goldberg (1976), where each of the 49 CPI scales in turn was predicted from varying numbers of PRF scales. The coefficients for the original CPI scales, plus $V_{0}$ and $P o$, are listed in Table 6. For six of the CPI scales (Dom, nDo, fAs, Do, Vo, and $P o$ ), the PRF regression equations should predict their values about as well as their scale reliabilities permit, with estimated correlations of approximately .80 . At the other extreme, for one CPI scale $(n N u)$ the equation should do quite poorly, probably no better than .30 . While for 
Table 4

Average Cross-Validity Coefficients as a Function of the Number of Predictors Included in the

Regression Equation: The Prediction of Each PRF Scale from the $49 \mathrm{CPI}$ Scales

\begin{tabular}{|c|c|c|c|c|c|}
\hline \multirow{2}{*}{$\begin{array}{c}\text { PRF } \\
\text { Scale } \\
\end{array}$} & \multicolumn{5}{|c|}{ Number of Predictors } \\
\hline & 1 & 2 & 3 & 4 & 5 \\
\hline$A b$ & .22 & .33 & .40 & .39 & $.44 \%$ \\
\hline$A c$ & .55 & .66 & .64 & $.66 *$ & .65 \\
\hline Af & $.64 \%$ & .61 & .61 & .63 & .62 \\
\hline $\mathrm{Ag}$ & .48 & .44 & .48 & $.52 *$ & .49 \\
\hline $\mathrm{Au}$ & .46 & .54 & .57 & .56 & $.58 \%$ \\
\hline $\mathrm{Ch}$ & .19 & .39 & .43 & .45 & $.46 *$ \\
\hline Cs & $.64 *$ & .61 & .63 & .60 & .60 \\
\hline $\mathrm{De}$ & .28 & .30 & .32 & .36 & $.37 \%$ \\
\hline Do & .79 & $.81 *$ & .80 & .80 & .79 \\
\hline En & .58 & .58 & .60 & $.60 *$ & .60 \\
\hline Ex & $.68 \%$ & .66 & .66 & .67 & .66 \\
\hline $\mathrm{Ha}$ & $.57 *$ & .52 & .56 & .53 & .52 \\
\hline Im & .48 & $.64 \%$ & .54 & .60 & .62 \\
\hline $\mathrm{Nu}$ & .50 & .64 & .63 & $.66 *$ & .65 \\
\hline $0 r$ & .75 & $.76 *$ & .75 & .73 & .72 \\
\hline $\mathrm{Pl}$ & .41 & .50 & .49 & .52 & $.53 \%$ \\
\hline $\mathrm{Se}$ & .07 & .08 & $.11 *$ & .10 & .09 \\
\hline $\mathrm{Sr}$ & .51 & .52 & .55 & .56 & $.57 *$ \\
\hline Su & .39 & .46 & .49 & .51 & $.52 \%$ \\
\hline Un & $.60 *$ & .58 & .53 & .50 & .53 \\
\hline In & - & - & - & - & - \\
\hline Dy & .65 & .65 & .65 & $.68 *$ & .67 \\
\hline Average & .49 & .53 & .54 & .55 & $.56 *$ \\
\hline
\end{tabular}

Note: $--N=77$ in each of the two subsamples.

* Highest value in each row.

Downloaded from the Digital Conservancy at the University of Minnesota, http://purl.umn.edu/93227. 


\section{Table 5}

Predicting the Average PRF Scale from Various Subsets of CPI Scales: Average Cross-Validity Coefficients as a Function of the Number of Predictors Included in the Regression Equation

\begin{tabular}{lccccc} 
& \multicolumn{5}{c}{ Number of Predictors } \\
\cline { 5 - 6 } Set of CPI Scales & 1 & 2 & 3 & 4 & 5 \\
\hline All 49 scales & .49 & .53 & .54 & .55 & .55 \\
11 Rational scales & .44 & .51 & .52 & .53 & .54 \\
11 Theoretical scales & .44 & .50 & .51 & .52 & .52 \\
18 Original scales & .40 & .47 & .50 & .49 & .50 \\
11 Factor scales & .39 & .42 & .44 & .44 & .45 \\
5 Rational scales & .36 & .41 & .43 & .44 & .44
\end{tabular}

Note:--Values are averaged across

eight CPI scales (Re, So, To, Ai. Py,fAm, fHa and $n D e$ ), the equations should only produce correlations around .45 ; for the remaining twothirds of the CPI scales, they should average around .65 .

\section{Stepwise Regresssion Analyses: Suggested Prediction Equations}

Table 7 presents the results from the stepwise regression analyses based on the total group of students, when the set of 49 CPI scales was used as an initial pool to predict each of 20 PRF scales. ${ }^{3}$ The decision on the optimal number of steps to include in each analysis was made first on the basis of the size (and statistical significance) of the increment in predictable variance in the analyses within the total

${ }^{3}$ Prediction equations using only the original CPI scales are presented in Goldberg (1976). These equations permit the estimate of only 13 PRF scale scores, in contrast to the 20 that are predictable when a larger set of CPI scales is scored.
21 PRF scales (In excluded).

group and secondly on the results from the corresponding cross-validity analyses (Table 4). For each equation, the CPI scales are listed in Table 7 in the order of their entry into the fullsample stepwise analyses. Included in the table are two estimates of the accuracy of each equation: (a) the multiple correlation in the total (derivation) sample, and (b) the average correlation for that particular number of steps from the cross-validity analyses (Table 4). The first index, the average of which across the 20 scales was .68 , is clearly an overestimate of expected cross-validity in new samples; the second index, which averaged .59 , is probably an underestimate. The provision of both an upper bound and a lower bound estimate permits an assessment of the likely range of values for these prediction equations in new samples.

The equations presented in Table 7 provide CPI predictions for 20 of the 22 PRF scales. Excluded from the table are equations for predicting the PRF Sentience $(S e)$ and Infrequency $(I n)$ scales. The latter, a validity scale 
Table 6

Average Cross-Validity Coefficients as a Function of the Number of Predictors Included in the Regression Equation: The Prediction of Each of 19 CPI Scales from the 22 PRF Scales

\begin{tabular}{llllll}
\hline CPI & \multicolumn{5}{c}{ Number of Predictors } \\
Scale & 1 & 2 & 3 & 4 & 5 \\
\hline Do & .74 & $.78 *$ & .77 & .77 & .77 \\
Cs & .22 & .48 & .49 & .55 & $.56 *$ \\
Sy & .56 & .65 & .65 & .65 & $.68 *$ \\
Sp & .56 & .56 & .62 & .64 & $.68 *$ \\
Sa & .55 & $.61 *$ & .55 & .57 & .52 \\
Wb & $.72 *$ & .72 & .69 & .71 & .71 \\
Re & .29 & .40 & $.40 *$ & .38 & .39 \\
So & .22 & .44 & .49 & $.51 *$ & .47 \\
Sc & .51 & .61 & .61 & .64 & $.65 *$ \\
To & .30 & $.48 *$ & .44 & .46 & .45 \\
Gi & .49 & .54 & .57 & .57 & $.61 *$ \\
Cm & - & - & - & - & - \\
Ac & .61 & $.67 *$ & .66 & .66 & .67 \\
Ai & .15 & .33 & .41 & $.44 *$ & .40 \\
le & $.56 *$ & .54 & .54 & .54 & .53 \\
Py & .18 & .30 & .38 & $.45 *$ & .43 \\
Fx & .53 & .59 & .67 & .65 & $.68 *$ \\
Fe & .42 & .55 & .54 & .57 & $.58 *$ \\
Vo & .69 & $.77 *$ & .75 & .76 & .75 \\
Po & .76 & .75 & .77 & .77 & $.77 *$ \\
\hline Average & .48 & .57 & .58 & .59 & $.59 *$ \\
\hline So & & & & & \\
\hline
\end{tabular}

Note: $--N=77$ in each of the two subsamples. * Highest value in each row. 


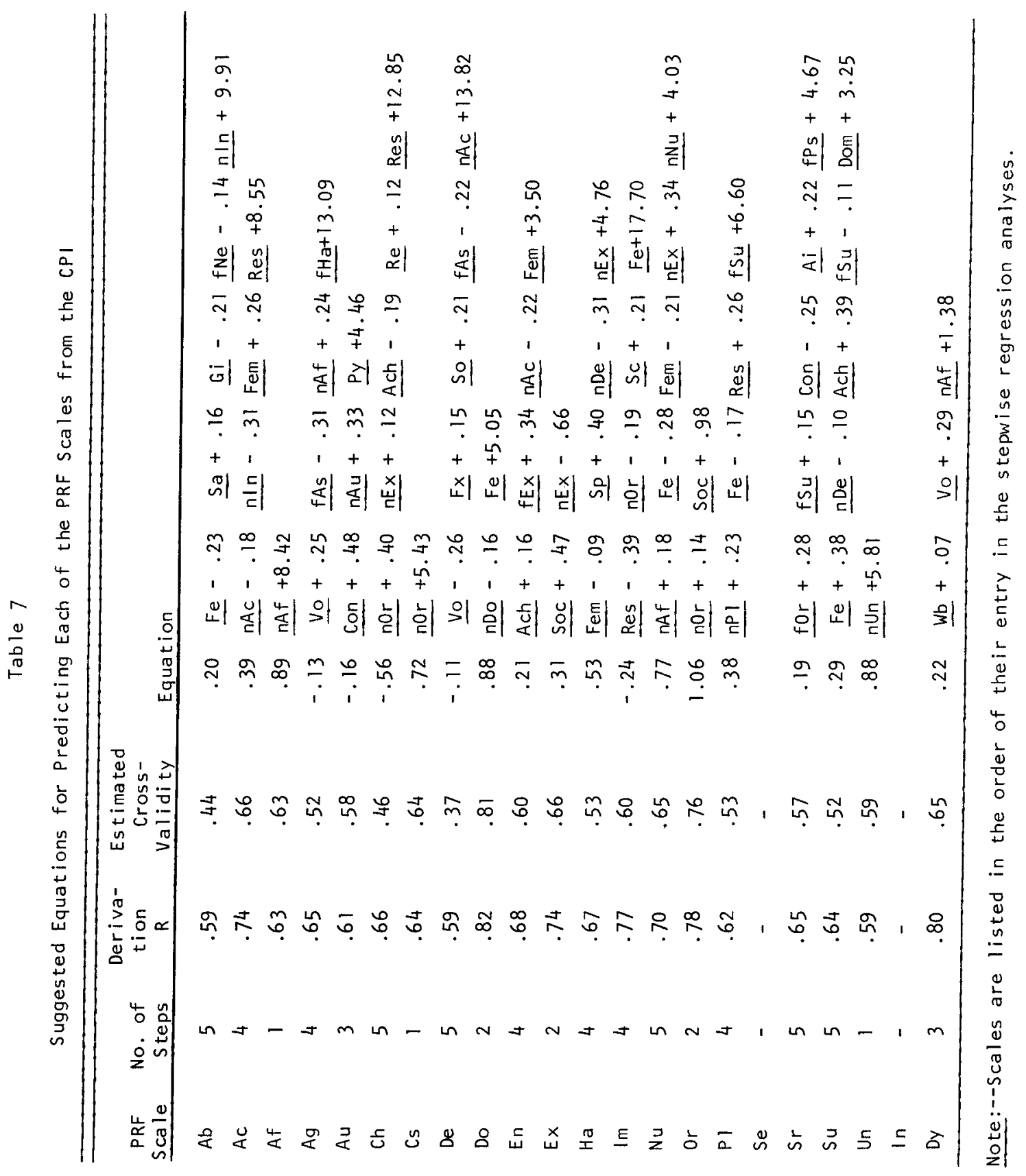


composed of highly improbable and statistically rare items (e.g., "I can run a mile in less than four minutes"), was used-with its CPI analogue, the Communality $(\mathrm{Cm})$ scale-to exclude deviant response protocols from the sample; as a consequence, the range of scores on $I n$ and $C m$ are severely attenuated. While validity scales like $I n$ and $C m$ are not reasonable candidates for cross-inventory prediction on purely methodological grounds, the PRF Sentience $(\mathrm{Se})$ scale, constructed to measure individual differences in sensuousness and openness to new experiences, simply measures one PRF content dimension that seems to be orthogonal to all of those provided by the CPI.

Table 8 presents PRF equations for predicting each of $19 \mathrm{CPI}$ scales, including all of the 18 original scales other than $C m$, plus $V o$ and $P o$. Since it is unlikely that investigators would wish to use the PRF to predict any of the CPI special scales (Hase \& Goldberg, 1967), equations for those scales have been omitted from this report (but are available from the author). Included in Table 8 are the same types of upper bound and lower bound cross-validity estimates as were presented in Table 7. For the average of the CPI original scales, these values were .68 and .58 respectively; when $V_{o}$ and $P_{o}$ are included in the set, the averages were .70 and .60 .

As Table 8 reveals, the typical CPI scale is predicted rather well by one or two PRF content scales and the Desirability $(D y)$ scale. $D y$ entered the stepwise regression analysis at the first step for nine CPI scales, at the second step for four others, and at the third step for three more. Indeed, the equations for only three of these $19 \mathrm{CPI}$ scales-Self-acceptance $(\mathrm{Sa})$, Flexibility $(F x)$, and Femininity $(F e)$ - do not include $D y$.

\section{A Final Word of Caution}

The future success of these equations as cross-inventory predictors will depend in part on the nature of the new groups in which they will be used. Specifically, predictive accuracy can be expected to drop in groups that differ substantially from the one used to derive the equations. As a consequence, until future investigations demonstrate the extent to which these equations are robust across diverse groups, it is wise to keep in mind the particular limitations of the group from which they were derived-specifically, its relatively small size $(N=154)$, its homogeneity in age and education (university undergraduates), and its informal test administration standards (the inventories having been administered under rather uncontrolled conditions). Hopefully, investigators with access to other groups will soon discover the effects of such limitations.

\section{References}

Anastasi, A. Review of the Personality Research Form. In O. K. Buros (Ed), Seventh mental measurements yearbook: Vol. 1. Highland Park, NJ: Gryphon Press, 1972. Pp. 297-298.

Campbell, J. B., \& Chun, K. Inter-inventory predictability and content overlap of the $16 \mathrm{PF}$ and the CPI. Applied Psychological Measurement. 1977, 1. 51-64.

Cattell, R. B., \& Bolton, L. S. What pathological dimensions lie beyond the normal dimensions of the 16PF? A comparison of MMPI and 16PF factor domains. Journal of Consulting and Clinical Psychology. 1969, 33, 18-29.

Cattell, R. B., Eber, H. W., \& Tatsuoka, M. M. Handbook for the Sixteen Personality Factor Questionnaire (16PF) in clinical. educational. in * dustrial and research psychology. Champaign, IL.: Institute for Personality and Ability Testing, 1970.

Edwards, A. L. The social desirability variable in personality assessment and research. New York: Dryden, 1957.

Goldberg, L. R. A historical survey of personality scales and inventories. In P. McReynolds (Ed.), Advances in psychological assessment: Vol. 2. Palo Alto, CA.: Science and Behavior Books, 1971. Pp. 293-336.

Goldberg, L. R. Parameters of personality inventory construction and utilization: A comparison of prediction strategies and tactics. Multivariate Behavioral Research Monograph. 1972, No. 722. (a) 


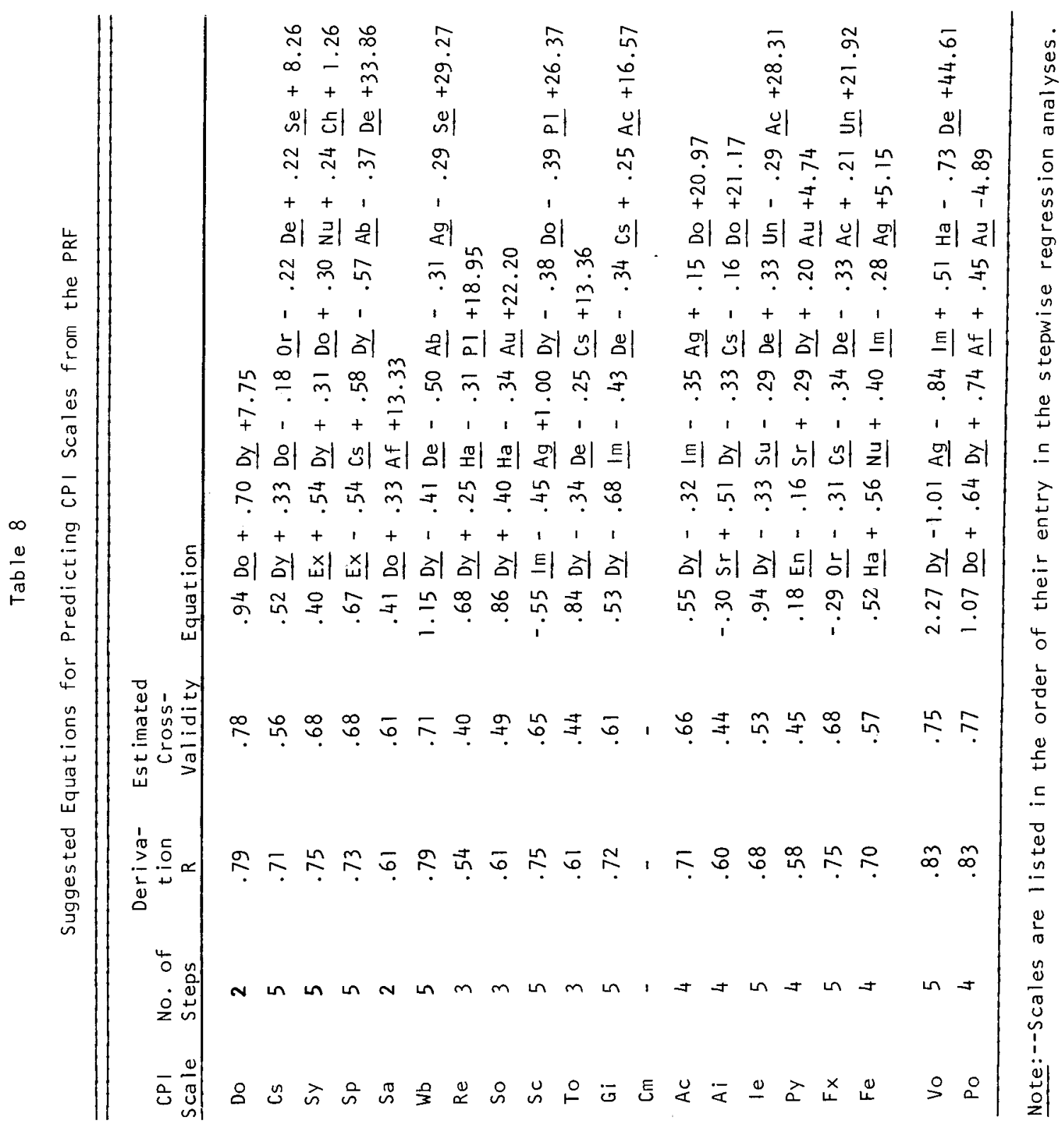


Goldberg, L. R. Review of the California Psychological Iventory. In O. K. Buros (Ed.), Seventh mental measurements yearbook: Vol. 1. Highland Park, N.J.: Gryphon Press, 1972. Pp. 94-96. (b)

Goldberg, L. R. What if we administered the "wrong" inventory? The prediction of scores on Personality Research Form scales from those on the California Psychological Inventory, and vice versa. Oregon Research Institute Research Bulletin. 1976, Vol. 16, No. 8.

Gough, H. G. Estimation of locus-of-control scores from the California Psychological Inventory, Psychological Reports. 1974, 35, 343-348.

Hase, H. D., \& Goldberg, L. R. Comparative validity of different strategies of constructing personality inventory scales. Psychological Bulletin. 1967, 67. 231-248.

Jackson, D. N. Stylistic response determinants in the California Psychological Inventory. Educational and Psychological Measurement. 1960, 20. 339-346.

Jackson, D. N. A sequential system for personality scale development. In C. D. Spielberger (Ed.), Current topics in clinical and community psychology: Vol. 2. New York: Academic Press, 1970. Pp. 61-96.

Jackson, D. N., \& Messick, S. Content and style in personality assessment. Psychological Bulletin. 1958, 55, 243-252.

Johnson, R. W., Flammer, D. P., \& Nelson, J. G. Multiple correlations between personality factors and SVIB occupational scales. Journal of Counseling Psychology. 1975, 22, 217-223.

Kelly, E. L. Review of the Personality Research Form. In O. K. Buros (Ed.), Seventh mental measurements yearbook: Vol. 1. Highland Park, NJ: Gryphon Press, 1972. Pp. 298-301.
Megargee, E. I. California Psychological Inventory handbook. San Francisco, CA: Jossey-Bass, 1972.

Murray, H. A., et al. Explorations in personality: A clinical and experimental study of fifty men of college age. New York: Oxford University Press, 1938.

Nesselroade, J. R., \& Baltes, P. B. Higher order factor convergence and divergence of two distinct personality systems: Cattell's HSPQ and Jackson's PRF. Multivariate Behavioral Research. 1975, 10, 387-407.

Nichols, R. C., \& Schnell, R. R. Factor scales for the California Psychological Inventory. Journal of Consulting Psychology. 1963, 27. 228-235.

Scott, D. P., \& Severance, L. J. Relationships between the CPI, MMPI, and locus of control in a nonacademic environment. Journal of Personality Assessment. 1975, 39. 141-145.

Stricker, L. J. Personality Research Form: Factor structure and response style involvement. Journal of Consulting and Clinical Psychology. 1974, 42. 529-537.

Wiggins, J. S. Review of the Personality Research Form. In O. K. Buros, (Ed.), Seventh mental measurements yearbook: Vol. 1. Highland Park, NJ: Gryphon Press, 1972. Pp. 301-303.

\section{Acknowledgements}

This project was supported by Grant No. MH12972 from the National Institute of Mental Health. U.S. Public Health Service.

\section{Author's Address}

Lewis R. Goldberg. Department of Psychology, University of Oregon, Eugene, Oregon 97403. 